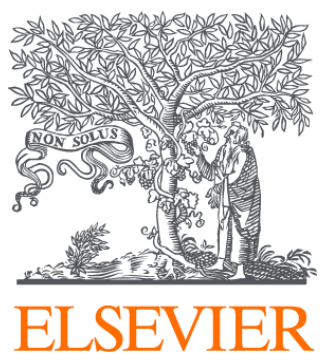

Since January 2020 Elsevier has created a COVID-19 resource centre with free information in English and Mandarin on the novel coronavirus COVID-

19. The COVID-19 resource centre is hosted on Elsevier Connect, the company's public news and information website.

Elsevier hereby grants permission to make all its COVID-19-related research that is available on the COVID-19 resource centre - including this research content - immediately available in PubMed Central and other publicly funded repositories, such as the WHO COVID database with rights for unrestricted research re-use and analyses in any form or by any means with acknowledgement of the original source. These permissions are granted for free by Elsevier for as long as the COVID-19 resource centre remains active. 


\title{
European ad-hoc consensus statement on gene replacement therapy for spinal muscular atrophy
}

\author{
Janbernd Kirschner ${ }^{\text {a, }}{ }^{\text {, }}$, Nina Butoianu ${ }^{\text {b }}$, Nathalie Goemans ${ }^{\text {c }}$, Jana Haberlova ${ }^{\text {, }}$ \\ Anna Kostera-Pruszczyk ${ }^{\mathrm{e}}$, Eugenio Mercuri ${ }^{\mathrm{g}}{ }^{\mathrm{h}}$, W. Ludo van der Pol ${ }^{\mathrm{k}}$, \\ Susana Quijano-Roy ${ }^{\mathrm{i}}$, Thomas Sejersen ${ }^{\mathrm{j}}$, Eduardo F. Tizzano ${ }^{\mathrm{f}}$, Andreas Ziegler ${ }^{1}$, \\ Laurent Servais $^{\mathrm{m}, \mathrm{n}, 1}$, Francesco Muntoni ${ }^{\mathrm{o}, 1}$ \\ a Department of Neuropediatrics, University Hospital Bonn, Bonn, Germany \\ b Pediatric Neurology Clinic, "Prof. Dr. Al. Obregia" Hospital, Bucharest, Faculty of Medicine and Pharmacy "Carol Davila”, Bucharest, Romania

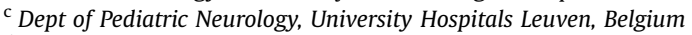 \\ d Dept of Pediatric Neurology, Motol University Hospital, Prague, Czech Republic \\ e Department of Neurology, Medical University of Warsaw, Poland \\ ${ }^{\mathrm{f}}$ Department of Clinical and Molecular Genetics, Medicine Genetics Group, University Hospital Vall d'Hebron, Barcelona, Spain \\ ${ }^{g}$ Pediatric Neurology, Università Cattolica del Sacro Cuore, Rome, Italy \\ ${ }^{\text {h }}$ Centro Clinico Nemo, Fondazione Policlinico Universitario Agostino Gemelli IRCCS, Rome, Italy \\ ${ }^{\mathrm{i}}$ Neuromuscular Unit, Child Neurology and ICU Department, Raymond Poincaré University Hospital (UVSQ), APHP Paris Saclay, Garches, France \\ ${ }^{\mathrm{j}}$ Department of Womeńs and Childreńs Health, Karolinska Institutet, Stockholm, Sweden \\ ${ }^{\mathrm{k}}$ Department of Neurology, UMC Utrecht Brain Center, University Medical Center Utrecht, Utrecht University, the Netherlands \\ ${ }^{1}$ Department of Neuropediatrics and Metabolic Medicine, Centre for Childhood and Adolescent Medicine, University Hospital Heidelberg, Germany \\ ${ }^{\mathrm{m}}$ University of Liège, Neuromuscular Reference Center Disease, Department of Pediatrics, Liege, Belgium \\ ${ }^{\mathrm{n}}$ MDUK Neuromuscular Center, Department of Pediatrics, University of Oxford, UK \\ ${ }^{\circ}$ Dubowitz Neuromuscular Centre, UCL Great Ormond Street Institute of Child Health, and NIHR Biomedical Research Centre, Great Ormond Street Hospital \\ for Children, London, UK
}

\section{A R T I C L E I N F O}

\section{Article history:}

Received 15 June 2020

Received in revised form

3 July 2020

Accepted 3 July 2020

\section{Keywords:}

Spinal muscular atrophy

Nusinersen

Onasemnogene abeparvovec

Zolgensma

Gene therapy

SMN1

SMN2

\begin{abstract}
A B S T R A C T
Spinal muscular atrophy (SMA) used to be one of the most common genetic causes of infant mortality. New disease modifying treatments have changed the disease trajectories and most impressive results are seen if treatment is initiated in the presymptomatic phase of the disease. Very recently, the European Medicine Agency approved Onasemnogene abeparvovec (Zolgensma ${ }^{\circledR}$ ) for the treatment of patients with SMA with up to three copies of the SMN2 gene or the clinical presentation of SMA type 1. While this broad indication provides new opportunities, it also triggers discussions on the appropriate selection of patients in the context of limited available evidence. To aid the rational use of Onasemnogene abeparvovec for the treatment of SMA, a group of European neuromuscular experts presents in this paper eleven consensus statements covering qualification, patient selection, safety considerations and longterm monitoring.
\end{abstract}

๑ 2020 European Paediatric Neurology Society. Published by Elsevier Ltd. All rights reserved.

\footnotetext{
* Corresponding author. Department of Neuropediatrics, University Hospital Bonn, Venusberg-Campus 1, Gb. 82, 53127, Bonn, Germany.

E-mail addresses: Janbernd.kirschner@ukbonn.de (J. Kirschner), nbutoianu91@ gmail.com (N. Butoianu), nathalie.goemans@uzleuven.be (N. Goemans), Jana. Haberlova@fnmotol.cz (J. Haberlova), anna.kostera-pruszczyk@wum.edu.pl (A. Kostera-Pruszczyk), eumercuri@gmail.com (E. Mercuri), W.L.vanderPol@ umcutrecht.nl (W.L. van der Pol), susana.quijano-roy@aphp.fr (S. Quijano-Roy), thomas.sejersen@ki.se (T. Sejersen), etizzano@vhebron.net (E.F. Tizzano), andreas ziegler@med.uni-heidelberg.de (A. Ziegler), laurent.servais@paediatrics.ox.ac.uk (L. Servais), f.muntoni@ucl.ac.uk (F. Muntoni).

${ }^{1}$ Both authors contributed equally to this work.
}

\section{Introduction}

Spinal muscular atrophy (SMA) is caused by autosomal recessive mutations of the SMN1 gene and is characterized by loss of motoneurons and progressive muscle weakness. The birth incidence of SMA is around 1 in 10,000 and it is thus classified as an orphan disease. Disease severity covers a broad spectrum and onset ranges from neonatal period to adulthood, while onset in the first years of live is most common [1]. A highly homologous gene, SMN2, does 
not lead to disease but acts as a disease modifier. Higher SMN2 copy numbers are associated with milder phenotypes [2]. During the last decade, drug development for SMA has made dramatic progress and changed the disease outcome for many patients. Nusinersen

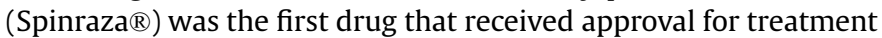
of SMA by the European Medicine Agency (EMA). Nusinersen is an antisense-oligonucleotide that is administered intrathecally and increases SMN protein concentration by modifying the splicing of the SMN2 gene. Another splicing modifier is Risdiplam, a small molecule that is administered orally and is still in clinical development [3].

An alternative treatment strategy for SMA is gene replacement therapy. Onasemnogene abeparvovec (formerly AVXS-101, Zolgensma $\left.{ }^{\circledR}\right)$ is an adeno-associated viral vector-based gene therapy designed to deliver a functional copy of the SMN1 gene to the motor neurons through a single intravenous infusion [4]. With a price around 2 million USD for a single dose, Zolgensma ${ }^{\circledR}$ has also attracted a great deal of attention as the most expensive drug on the market. In 2019 FDA approved the intravenous administration for the treatment of patients with SMA in the first two years of life independent of their disease severity. Recently, the EMA also approved Zolgensma ${ }^{\circledR}$ for the treatment of SMA in Europe. The label covers two overlapping groups of patients, but does not define any age or weight limit: [5].

- Patients with 5q spinal muscular atrophy (SMA) with a bi-allelic mutation in the SMN1 gene and a clinical diagnosis of SMA Type 1

- Patients with 5q SMA with a bi-allelic mutation in the SMN1 gene and up to 3 copies of the SMN2 gene.

According to this broad label a large number of patients with SMA would theoretically be eligible for treatment with Zolgensma ${ }^{\circledR}$. However, available data from clinical trials cover only patients during the first six months of life with a weight below $8.4 \mathrm{~kg}$ and little is known about the safety and efficacy of Zolgensma ${ }^{\circledR}$ in older or heavier patients. This discrepancy poses major challenges to patients, clinicians and payers associated with the question who should be treated under which circumstances, also in view of the fact that an effective and safe treatment, nusinersen, is already widely available in this patient population. While we acknowledge the need of a sound risk-benefit assessment for each individual patient and the diversity of societies and health systems across Europe, we also strongly believe that some general principles should be considered in these reflections. This ad-hoc consensusbased opinion statement aims to facilitate this process.

\section{Methodology}

This consensus statement has been developed by a group of neuromuscular experts from a variety of countries across Europe. The size and composition of the group was determined to allow a broad representation but also to facilitate a consensus within an acceptable time frame. This was of particular importance as discussions about the indication and reimbursement of Zolgensma ${ }^{\circledR}$ have already started in individual countries.

Given the time frame and the fact that all participating experts are very familiar with the limited published data on the use of Zolgensma ${ }^{\circledR}$ for the treatment of SMA, and of the data on other approved therapeutic options, we decided that a systematic literature review was not necessary in advance of the consensus process.

Due to travel restrictions associated with the COVID-19 pandemic, an in-person meeting was not possible. To initiate the process, all co-authors participated in a two-hour video conference to agree on the consensus process and to identify potential areas where a consensus statement might be helpful. The areas of interest were then further specified in a shared online document and interactive discussion during a period of two weeks. After general consensus on the appropriate statements, we performed an anonymous voting with all experts on each statement using REDCap electronic data capture tools. With this final vote we aimed to avoid dominating influence of some experts and allow free expression of opinions. A consensus greater than $95 \%$ was considered "strong consensus", between 75 and 95\% "consensus", and between 50 and $75 \%$ "majority consensus". If less than $50 \%$ approved a statement, it was labelled as "no consensus".

\section{Consensus statements}

\subsection{Selection criteria for gene therapy}

Consensus statement 1: Traditional SMA types (e.g. type 0, 1, 2, $3,4)$ alone are not sufficient to define patient populations who might benefit most from gene therapy. In symptomatic patients age at onset, disease duration and motor function status at the start of treatment are the most important factors that predict response to treatment.

\section{- Strength of consensus: Strong consensus (100\%)}

Rationale: SMA represents a continuous spectrum of disease severity. The traditional classification is based on disease onset and the maximal motor milestone acquired. However, there is significant overlap between the different types. As SMA is a progressive disease, the clinical status of an individual patient does not only depend on the type of SMA but also on the stage of the disease. For example, the clinical condition of a patient with severe SMA type 2 in advanced stages of disease can be significantly more severe compared with a patient in early stages of SMA type 1. In addition, since the introduction of disease-modifying treatments, several patients originally belonging to type 1 or type 2 have acquired sitting position or ambulation, respectively [6], and thus cross the boundaries of the traditional classification. In fact, disease stage and duration might be more important predictors of outcome than the subtype of SMA. Therefore, traditional SMA types alone are not sufficient to characterize individual patients and one should consider additional factors to define populations that might benefit most from gene replacement and other disease-modifying treatments.

Consensus statement 2: In presymptomatic patients SMN2 copy number is the most important predictor of clinical severity and age of onset. As long as no better biomarkers or predictors are available, treatment decisions for presymptomatic patients should primarily be based on SMN2 copy number. Determination of SMN2 copy number needs to be performed in an expert laboratory with adequate measures of quality control.

\section{- Strength of consensus: Strong consensus (100\%)}

Rationale: The growing evidence that initiation of diseasemodifying treatments in the presymptomatic stages of SMA is associated with significantly better outcome [7] leads to an increasing number of newborn screening programs and patients who are diagnosed before they develop any symptoms [8]. As the traditional classification of SMA is based on clinical symptoms, it is not applicable in the presymptomatic stages of SMA. Currently, SMN2 copy number is the best available predictor of disease severity, even if limitations of the predictive value remain [2,9]. Work is underway to identify additional biomarkers, such a 
phosphorylated neurofilaments, but none has so far reached either the sufficient robustness, either the current clinical practice [8]. Determination of SMN2 copy numbers is not trivial, and discordant results have been reported between different methods and laboratories [10]. Therefore, appropriate quality control measures are indispensable, especially when SMN2 copy numbers are used for treatment decisions in presymptomatic patients with SMA.

Consensus statement 3: Approval of gene therapy for SMA with Zolgensma ${ }^{\circledR}$ is based on clinical trials with patients with SMA less than 6 months of age. Additional data of patients up to 2 years and weighing up to $13.5 \mathrm{~kg}$ are made public through congress presentations. These data mainly come from non-systematic data collection in the US, where Zolgensma $\mathbb{R}$ is approved up to the age of 2 years. When administered after the age of 6 months and/or in advanced stages of the disease, parents or patients should clearly be made aware that there are so far no published data on efficacy and safety. In this patient population it is particularly important for physicians to discuss the benefit/risk ratio and to carefully manage parents' or patients' expectations.

\section{- Strength of consensus: Strong consensus (100\%)}

Rationale: The broad label for Zolgensma ${ }^{\circledR}$ clearly exceeds the age and weight limits that have been studied in clinical trials and does not imply that its use is effective and safe in all patients who meet the criteria. All clinical trials excluded patients in advanced stages of the disease. The European Medicine Agency deployed a conditional marketing authorisation, which can be used if a medicine addresses an unmet medical need and the benefit of immediate availability outweighs the risk from less comprehensive data than normally required. Growing evidence suggests that early initiation of treatment in a less advanced disease stage is associated with better outcome $[11,12]$. As the applied dose is proportional to the patient's body weight, treatment of heavier patients also implies a significantly higher total dose than previously used in clinical trials. It is possible that advanced disease stage and higher total dose have a negative impact on the risk-benefit ratio. Treating physicians, patients and families should be aware of these uncertainties, and also discuss other approved therapies in which the risk-benefit might be better known.

Consensus statement 4: In patients presenting symptoms at birth, treated after a long disease duration, or with already severe evolution, parents should be clearly made aware that despite the use of gene therapy there is a high risk of living with a very severe disability. Palliative care should be discussed as an alternative treatment option in these circumstances.

\section{- Strength of consensus: Strong consensus (100\%)}

Rationale: All disease modifying therapies for SMA have demonstrated a better efficacy when administered early [11]. Patients treated with Zolgensma ${ }^{\circledR}$ who present with the most impressive evolution are patients treated before symptom onset or with a very short disease duration. This is exemplified by circulating videos of individual patients with SMA type 1 achieving the ability to walk and climb stairs at a young age. This improvement is exceptional in symptomatic children with SMA type 1 and may be misleading both for some parents of much more affected children, and for clinicians who are not deeply involved in this field of research. For treatment with Nusinersen Aragon-Gawinska et al. have shown that higher baseline motor function is associated with higher probability of acquisition of motor milestones [13]. At the other end of the spectrum, more severely affected patients, who do already depend on respiratory support and tube feeding at initiation of treatment, do in most cases only demonstrate very modest improvement if at all [14-16]. In these severe cases, gene replacement therapy and other disease modifying treatments might stabilize the disease but not necessarily reduce disability or improve quality of life.

Consensus statement 5: Since the risk of gene therapy increases with the dose administered and since the dose is directly proportional with the weight, patients above $13.5 \mathrm{~kg}$ should only be treated in specific circumstances. For these patients, treatment with other disease modifying therapies or future intrathecal administration of Zolgensma ${ }^{\circledR}$ should be considered as an alternative.

\section{- Strength of consensus: Strong consensus (100\%)}

Rationale: The larger amounts of vector needed to treat patients with higher body weight cause concerns about potential side effects associated with the immunological response to peripheral transduction of other organs. Intrathecal administration is therefore being explored as an alternative route of administration. In mice and nonhuman primates intrathecal administration of AAV9 led to widespread transgene expression throughout the spinal cord when using significantly lower doses compared to the intravenous application [17]. The intrathecal administration of Zolgensma ${ }^{\circledR}$ is subject of a clinical trial for patients with SMA type 2 between 6 and 60 months of age (NCT03381729). This study is currently on clinical hold due to pre-clinical findings of dorsal ganglia damage in non-human primates. It remains to be seen if intrathecal dosing might be associated with a better risk-benefit ratio in older patients. In this context it should also be noted, that previous intravenous treatment with Zolgensma ${ }^{\circledR}$ will immunize patients against the vector and thus most likely preclude future intrathecal administration. In addition, nusinersen is an approved drug which is also available as an alternative treatment and has been studied in a double-blind placebocontrolled trial in later-onset types of SMA [18]. Several recent manuscripts also address the efficacy and safety of nusinersen in the real world setting, broadly confirming the observation from the original phase 3 studies, and extending the age range of patients in whom the role of this drug has been explored [19-23].

Consensus statement 6: Until now there is no published evidence that combination of two disease modifying therapies (e.g. gene therapy and nusinersen) is superior to any single treatment alone.

\section{- Strength of consensus: Strong consensus (100\%)}

Rationale: SMN1 gene therapy and splicing modifiers for SMN2 both exert their action through an increase of SMN protein [3]. Head to head studies comparing the amount of SMN protein expression or clinical effect size are not available. The combination of both approaches has also not been studied systematically and warrants further investigation [24]. However, from a theoretical point of view one would not expect the combinational effect to be equal to the sum of their single effects due to the common downstream pathway and mode of action, unless the biodistribution of the different therapeutic compounds was substantially different. Before more evidence is available, combination of both approved therapies should not be part of routine care. In severe symptomatic patients, irreversible degeneration of motor neurons and muscle tissue are probably the most important factors for any lack of efficacy or rescue of the phenotype regardless of the (higher) amount of SMN protein available from any treatment. 


\subsection{Structural requirements for administration of gene therapy}

Consensus statement 7: Centres performing gene therapy for SMA should have broad expertise in the assessment and treatment of SMA according to international standards. They should also have the ability and resources to deal with potential side effects of gene therapy. Personnel should be trained and have experience in the use of standardized and validated outcome measure for SMA to document treatment effects. Recognition as European Reference Centre (www.ern-euro-nmd.eu) or national accreditation as neuromuscular centre of expertise might serve as additional selection criteria.

\section{- Strength of consensus: Strong consensus (100\%)}

Rationale: Zolgensma ${ }^{\circledR}$ is the first approved gene therapy for neuromuscular diseases. As outlined above selection of appropriate patients is challenging and requires comprehensive knowledge of the clinical presentation of SMA, available treatment options and potential risks associated with the use of gene therapy. To ensure appropriate monitoring and to generate more robust evidence, treatment centres should use established and standardized outcome measures [25]. As most patients will remain with significant disease burden even after application of gene therapy, treatment centres should also be capable to provide appropriate multidisciplinary care according to international consensus recommendations $[1,26]$. National health systems need to ensure that treatment centres are appropriately qualified and provided with sufficient resources to implement, monitor and evaluate the use of gene therapy for SMA in the best possible manner.

Consensus statement 8: There is convincing evidence that early initiation of treatment - ideally in the presymptomatic stage of the disease - is associated with markedly better outcome as compared to later start of treatment. Spinal Muscular Atrophy is therefore a good candidate for inclusion in newborn screening programs. In newly diagnosed patients any delay of treatment should be avoided. Ideally, the time frame between diagnosis and initiation of a disease modifying treatment should be no longer than 14 days. This is particularly important in infants due to the progressive course of the disease.

\section{- Strength of consensus: Strong consensus (100\%)}

Rationale: Published and unpublished data from clinical trials and newborn screening programs indicate that presymptomatic initiation of treatment is often associated with normal motor development during infancy $[7,27,28]$. In addition, several studies indicate that age and functional status at initiation of treatment are key predictors for the effect size of disease modifying treatments [14-16,27,29,30]. Especially early-onset SMA is a rapidly progressing disease in which the clinical status can deteriorate within a week. In analogy to the saying "time is brain" for the treatment of stroke [31] one could coin the phrase "time is motoneuron" for the treatment of early-onset SMA. Several factors can contribute to a potential delay of treatment initiation and include clinical diagnosis, genetic confirmation, drug availability and reimbursement decisions. During this time it is also important to establish a trusting and confident communication with the parents, who are often confronted with a new diagnosis and need to be involved in shared decision making about any potential treatment. Thus, a joined effort is needed to facilitate timely initiation of treatment if deemed appropriate.

\subsection{Generation of additional evidence}

Consensus statement 9: Data concerning effectiveness and safety should be collected systematically for all patients treated. Treatment centres should be provided with adequate resources to perform long-term monitoring of treated patients with standardized outcome measures. Where available disease specific registries should be used for data collection to allow comparison between different treatments. Data analysis should be performed primarily by academic institutions and networks.

\section{- Strength of consensus: Strong consensus (100\%)}

Rationale: As often inevitable with orphan diseases, approval of Zolgensma ${ }^{\circledR}$ for the treatment of SMA is based on limited data. Available clinical trials cover only a subgroup of patients mostly in the early stages of the disease and with a short observation period. Although real-world data collections can never reach the internal validity of controlled clinical trials, they can significantly contribute to evaluate the long-term effectiveness and safety of gene therapy for SMA. Considering the existing evidence gaps and the financial burden associated with gene replacement therapy, long-term follow up data should be collected for all patients treated with Zolgensma ${ }^{\circledR}$. As this follow-up is often not part of an interventional clinical trial but routine care, adequate and sustainable funding needs to be allocated through the health system. Ideally, data collection should be performed in disease specific registries with shared datasets, that will also allow comparison of different disease modifying treatments. Data ownership, analysis and publication by academic institutions and networks should help to reduce any potential commercial bias [32-34].

Consensus statement 10: On the basis of the currently available data and in light of existing effective treatment alternatives, intravenous gene replacement therapy with Zolgensma ${ }^{\circledR}$ for patients with a body weight $>13.5 \mathrm{~kg}$ should only be performed under a more rigorous protocol with continuous monitoring of safety and efficacy. This data collection might be best achieved in a clinical trial setting.

\section{- Strength of consensus: Strong consensus (100\%)}

Rationale: Currently there is no experience with the intravenous use of Zolgensma $₫$ in patients with a body weight above $13,5 \mathrm{~kg}$ and it might be associated with additional risks due to the high amount of viral vector. If the use of gene therapy for this population is deemed appropriate, it should only be performed under a rigorous protocol and close safety monitoring, and after having carefully considered the other approved therapeutic options. An interventional clinical trial might be the most appropriate setting to achieve this.

Consensus statement 11: As the use of Zolgensma ${ }^{\circledR}$ will generate additional evidence during the coming years, pharmaceutical industry, regulators, patient representatives, and academic networks should collaborate to ensure that any new data on effectiveness and safety are publicly available in an unbiased and timely manner. This growing body of evidence is indispensable for an improved risk-benefit assessment for future patients and should not be hampered by particular commercial or academic interests.

\section{- Strength of consensus: Strong consensus (100\%)}

Rationale: Currently the number of subjects exposed to gene therapy with Zolgensma $\otimes$ is still limited. Ongoing clinical trials and 
real-word experience will generate additional evidence including long-term effects. However, the fact that the data on safety and effectiveness are collected by different institutions such as pharmaceutical company, regulatory authorities and academic networks might impede to generate an integrated body of evidence in a timely manner. All contributing parties should make these data available in an unbiased manner. Identification of new safety signals is specifically important for orphan drugs where the experience is still limited at the time of approval [35]. Therefore, any new safety signal should not only be communicated with clinical trial sites but with all treatment centres.

\section{Declaration of competing interest}

For the work presented here the authors did not receive any financial or other material support. Outside the submitted work authors declare the following potential conflicts of interest.

Janbernd Kirschner received honoraria for participation in advisory boards and educational activities from Avexis, Biogen, Roche, and ScholarRock. He received funding for clinical research from Biogen and AveXis.

Nina Butoianu - nothing to disclose.

Nathalie Goemans: participated in ad hoc advisory board activities for Biogen, Avexis, Roche and has received speaker's fee for participation in symposia from Roche and Biogen.

Jana Haberlova participated in advisory board activities and has received speaker's fee for participation in symposia for Biogen, Novartis and Roche.

Anna Kostera-Pruszczyk reports advisory board for Biogen, Avexis/Novartis, Roche, PTC, and grant support from Biogen, travel support from Biogen and Roche.

Eduardo F. Tizzano reports grant support to conduct clinical trials on SMA from Ionis/Biogen. Serves as a consultant to AveXis, Biogen, Biologix, Cytokinetics, Roche for participation to scientific advisory boards and participation in educational symposia.

Eugenio Mercuri reports acting as a consultant for AveXis, Biogen, Roche, Novartis, Cytokinetics and Scholar Rock; receiving honoraria from AveXis, Biogen, Roche, for participation to scientific advisory boards and participation in educational symposia; receiving investigator grant from Biogen for the iSMAc disease registry; acting as Principal investigator for Ionis/Biogen nusinersen trials; Roche olesoxime and risdiplam trial, Scholar Rock and Avexis zolgesma trials, all in SMA.

Susana Quijano-Roy participated in and received honoraria for lectures and advisory board activities for Biogen, Avexis, and Roche.

Thomas Sejersen: participated in and received honoraria for lectures and advisory board activities for Biogen, Avexis, and Roche.

W. Ludo van der Pol participated in ad hoc advisory board activities for Biogen and Avexis and served as a member of the Data Monitoring Committee for Branaplam (Novartis). Principal investigator for Jewelfish (Roche) and Topaz studies (Scholar Rock) and member of the scientific advisory board for SMA Europe.

Andreas Ziegler reports personal fees and scientific funding from Biogen, personal fees from Avexis, outside the submitted work.

Laurent Servais reports research support from AveXis, Roche and Biogen, and consultancy fees/board member of Avexis, Biogen, Roche and Cytokinetics.

Francesco Muntoni reports serving on the Pfizer Rare Diseases advisory board; acting as a consultant for AveXis, Biogen, Roche, Novartis; receiving honoraria from AveXis, Biogen, Roche, for participation to scientific advisory boards and participation in educational symposia; receiving investigator grant from Biogen for SMA REACH UK registry; acting as Principal investigator for Ionis/ Biogen nusinersen trials; Roche olesoxime and risdiplam trial and Avexis zolgesma trials, all in SMA.

\section{Acknowledgement}

We would like to thank Adrian Tassoni and Johanna Andrae from the Clinical Trials Unit of the Medical Center - University of Freiburg, Germany, for setting up the anonymous online voting.

\section{References}

[1] E. Mercuri, R.S. Finkel, F. Muntoni, et al., Diagnosis and management of spinal muscular atrophy: Part 1: recommendations for diagnosis, rehabilitation, orthopedic and nutritional care, Neuromuscul. Disord. 28 (2018) 103-115.

[2] M. Calucho, S. Bernal, L. Alias, et al., Correlation between SMA type and SMN2 copy number revisited: an analysis of 625 unrelated Spanish patients and a compilation of 2834 reported cases, Neuromuscul. Disord. 28 (2018) 208-215.

[3] D.C. Schorling, A. Pechmann, J. Kirschner, Advances in treatment of spinal muscular atrophy - new phenotypes, new challenges, new implications for care, J. Neuromuscul. Dis. 7 (2020) 1-13.

[4] J.R. Mendell, S. Al-Zaidy, R. Shell, et al., Single-dose gene-replacement therapy for spinal muscular atrophy, N. Engl. J. Med. 377 (2017) 1713-1722.

[5] European Medicine Agency, Zolgensma Marketing Authorisation. https:// www.ema.europa.eu/en/medicines/human/EPAR/zolgensma.

[6] T. Gidaro, L. Servais, Nusinersen treatment of spinal muscular atrophy: current knowledge and existing gaps, Dev. Med. Child Neurol. 61 (2019) 19-24.

[7] D.C. De Vivo, E. Bertini, K.J. Swoboda, et al., Nusinersen initiated in infants during the presymptomatic stage of spinal muscular atrophy: interim efficacy and safety results from the Phase 2 NURTURE study, Neuromuscul. Disord. 29 (2019) 842-856.

[8] T. Dangouloff, A. Burghes, E.F. Tizzano, L. Servais, N.S.S. Group, 244th ENMC international workshop: newborn screening in spinal muscular atrophy May 10-12, 2019, Hoofdorp, The Netherlands, Neuromuscul. Disord. 30 (2020) 93-103.

[9] A. Saffari, S. Kolker, G.F. Hoffmann, M. Weiler, A. Ziegler, Novel challenges in spinal muscular atrophy - how to screen and whom to treat? Ann. Clin. Transl. Neurol. 6 (2019) 197-205.

[10] D.C. Schorling, J. Becker, A. Pechmann, T. Langer, B. Wirth, J. Kirschner, Discrepancy in redetermination of SMN2 copy numbers in children with SMA, Neurology 93 (2019) 267-269.

[11] T. Dangouloff, L. Servais, Clinical evidence supporting early treatment of patients with spinal muscular atrophy: current perspectives, Therapeut. Clin. Risk Manag. 15 (2019) 1153-1161.

[12] L.P. Lowes, L.N. Alfano, W.D. Arnold, et al., Impact of age and motor function in a phase $1 / 2 \mathrm{~A}$ study of infants with SMA type 1 receiving single-dose gene replacement therapy, Pediatr. Neurol. 98 (2019) 39-45.

[13] K. Aragon-Gawinska, A. Daron, A. Ulinici, et al., Sitting in patients with spinal muscular atrophy type 1 treated with nusinersen, Dev. Med. Child Neurol. 62 (2020) 310-314

[14] A. Pechmann, T. Langer, D. Schorling, et al., Evaluation of children with SMA type 1 under treatment with nusinersen within the expanded access program in Germany, J. Neuromuscul. Dis. 5 (2018) 135-143.

[15] M.A. Farrar, H.L. Teoh, K.A. Carey, et al., Nusinersen for SMA: expanded access programme, J. Neurol. Neurosurg. Psychiatry 89 (2018) 937-942.

[16] S. Messina, M. Pane, V. Sansone, et al., Expanded access program with Nusinersen in SMA type I in Italy: strengths and pitfalls of a successful experience, Neuromuscul. Disord. 27 (2017) 1084-1086.

[17] K. Meyer, L. Ferraiuolo, L. Schmelzer, et al., Improving single injection CSF delivery of AAV9-mediated gene therapy for SMA: a dose-response study in mice and nonhuman primates, Mol. Ther. 23 (2015) 477-487.

[18] E. Mercuri, B.T. Darras, C.A. Chiriboga, et al., Nusinersen versus sham control in later-onset spinal muscular atrophy, N. Engl. J. Med. 378 (2018) 625-635.

[19] F. Audic, M.G.G. de la Banda, D. Bernoux, et al., Effects of nusinersen after one year of treatment in 123 children with SMA type 1 or 2: a French real-life observational study, Orphanet J. Rare Dis. 15 (2020) 148.

[20] T. Hagenacker, C.D. Wurster, R. Gunther, et al., Nusinersen in adults with $5 q$ spinal muscular atrophy: a non-interventional, multicentre, observational cohort study, Lancet Neurol. 19 (2020) 317-325.

[21] M.C. Walter, S. Wenninger, S. Thiele, et al., Safety and treatment effects of nusinersen in longstanding adult 5q-SMA type 3 - a prospective observational study, J. Neuromuscul. Dis. 6 (2019) 453-465.

[22] M. Pane, G. Coratti, V.A. Sansone, et al., Nusinersen in type 1 spinal muscular atrophy: twelve-month real-world data, Ann. Neurol. 86 (2019) 443-451.

[23] A. Veerapandiyan, K. Eichinger, D. Guntrum, et al., Nusinersen for older patients with spinal muscular atrophy: a real-world clinical setting experience, Muscle Nerve 61 (2020) 222-226.

[24] B.H. Lee, E. Collins, L. Lewis, et al., Combination therapy with nusinersen and AVXS-101 in SMA type 1, Neurology 93 (2019) 640-641.

[25] J. Montes, A.M. Gordon, S. Pandya, D.C. De Vivo, P. Kaufmann, Clinical outcome measures in spinal muscular atrophy, J. Child Neurol. 24 (2009) 968-978.

[26] R.S. Finkel, E. Mercuri, O.H. Meyer, et al., Diagnosis and management of spinal muscular atrophy: Part 2: pulmonary and acute care; medications, supplements and immunizations; other organ systems; and ethics, Neuromuscul. Disord. 28 (2018) 197-207.

[27] Care IfQaEiH, Newborn Screening for 5q-Linked Spinal Muscular Atrophy 
IQWiG Reports - Commission No. S18-02. Cologne (Germany), 2020.

[28] K. Vill, H. Kolbel, O. Schwartz, et al., One year of newborn screening for SMA results of a German pilot project, J. Neuromuscul. Dis. 6 (2019) 503-515.

[29] K. Aragon-Gawinska, A.M. Seferian, A. Daron, et al., Nusinersen in patients older than 7 months with spinal muscular atrophy type 1: a cohort study, Neurology 91 (2018) e1312-e1318.

[30] M. Pane, C. Palermo, S. Messina, et al., Nusinersen in type 1 SMA infants, children and young adults: preliminary results on motor function, Neuromuscul. Disord. 28 (2018) 582-585.

[31] M.D. Hill, V. Hachinski, Stroke treatment: time is brain, Lancet 352 (Suppl 3) (1998) SIII10-14.

[32] E. Mercuri, R. Finkel, M. Scoto, et al., Development of an academic disease registry for spinal muscular atrophy, Neuromuscul. Disord. 29 (2019) 794-799.

[33] A. Pechmann, K. Konig, G. Bernert, et al., SMArtCARE - a platform to collect real-life outcome data of patients with spinal muscular atrophy, Orphanet J. Rare Dis. 14 (2019) 18.

[34] H. Lochmuller, D. Evans, W. Farwell, et al., Position statement: sharing of clinical research data in spinal muscular atrophy to accelerate research and improve outcomes for patients, J. Neuromuscul. Dis. 5 (2018) 131-133.

[35] M. Sardella, C. Lungu, Evaluation of quantitative signal detection in EudraVigilance for orphan drugs: possible risk of false negatives, Ther. Adv. Drug Saf. 10 (2019), 2042098619882819. 